\title{
Two-year follow-up after holmium laser enucleation of the prostate and bipolar transurethral resection of the prostate: a prospective randomized study
}

\author{
Mamdouh M. El-Hawy ${ }^{1}$, Amr Eldakhakhny², Ahmed AbdEllatif ${ }^{3}$,Emad A. Salem ${ }^{4}$, Ahmed Ragab \\ Mostafa S. Elsharkawy ${ }^{1}$, Mostafa M. Abdelghani ${ }^{1}$, Luay Alshara ${ }^{5}$, Mohamed G. Hasanein ${ }^{1}$, Ahmed H. Ismail', \\ Ebrahim M. Ismail ${ }^{4}$, Mohamed A. Hassan ${ }^{1}$ and Ahmed I. Ali ${ }^{1 *}$
}

\begin{abstract}
Background: Different treatment options are available for the management of BPH. Our study aimed to compare the surgical outcomes of a holmium laser enucleation of the prostate (HoLEP) and a bipolar transurethral resection of the prostate (Bipolar-TURP) after 2 years.

Methods: Our prospective randomized study included 114 patients: 55 patients underwent HoLEP procedure and 59 patients underwent bipolar TURP procedure. All patients underwent a complete preoperative assessment and a physical examination. The postoperative follow-up data included $Q$ max and IPSS recordings at 1, 3, 6, 12, and 24 months and PVR urinary volume recordings at 6 and 12 months. Any postoperative complications were also recorded.
\end{abstract}

Results: There were no statistically significant differences between both groups regarding IPSS and Q max scores at one and 24 months postoperative. Also, there were no statistically significant differences between both groups regarding postoperative PVR at 6 and 12 months. One patient in the HoLEP group developed total incontinence after surgery.

Conclusion: Our study did not show a significant difference between HoLEP and bipolar TURP regarding postoperative $Q$ max and IPSS scores at 24 months of follow-up.

Keywords: BPH, HoLEP, Bipolar, TURP

\section{Background}

Benign prostatic hyperplasia (BPH) is a common disease affecting men over the age of 60 years. BPH can considerably increase patients' morbidity and worsen their quality of life. Different treatment options can be offered to BPH patients, including observation, pharmacological treatment, minimally invasive procedures, and open surgery.

\footnotetext{
*Correspondence: ahmed_issam79@yahoo.com

1 Department of Urology, School of Medicine, Minia University,

Minya 61111, Egypt

Full list of author information is available at the end of the article
}

Alpha-blockers and 5-ARIs are the primary medications used to treat $\mathrm{BPH}[1]$.

While surgical management of BPH is always debatable, for many years, the monopolar transurethral resection of the prostate (monopolar-TURP) has been accepted as the gold standard to surgically alleviate obstructive voiding dysfunction in men with BPH [2]. However, over the past decade, consistent data have demonstrated the superiority of other means of surgical intervention, and bipolar TURP and a holmium laser enucleation of the prostate (HoLEP) are considered well-established surgical treatments for $\mathrm{BPH}[3]$. 
Since its introduction, bipolar TURP has gained exceptional popularity among urologists. The bipolar resection of BPH allows for a resection with saline solution, achieving the advantage of improved hemostasis and a lower risk of TURP syndrome [4]. However, there are concerns about the bipolar current causing increased incidence of urethral stricture [5].

Rapid advances in technology have ensured that lasers will play an important role in the surgical management of $\mathrm{BPH}$ [6]. Gilling et al. first reported the holmium laser resection of the prostate (HoLRP) for treatment of $\mathrm{BPH}$ [7]. HoLRP has evolved into HoLEP with intravesical prostatic tissue morcellation.

The HoLEP procedure is a retrograde dissection of the prostatic lobes from the surgical capsule. Once enucleation performed, the tissue can be removed from the bladder by the morcellator. Different randomized trials comparing the HoLEP and TURP procedures have shown that HoLEP is associated with lower transfusion rates, shorter hospital stays, and shorter catheter times than the TURP procedure [8].

Our study aimed to compare the surgical outcomes of the two procedures in not only the early postoperative period but throughout a 24-month follow-up period.

\section{Methods}

Our study is a prospective randomized study conducted between January 2015 and January 2017, and local ethical committee approval was obtained. The study enrolled 124 patients who had presented to the clinic with symptomatic BPH and met our inclusion criteria (Fig. 1).

All patients signed consents before participation in the study. Of the 124 patients enrolled, 114 patients completed the study.

The inclusion criteria were bothersome urinary symptoms not responding to medical treatment with alpha blockers; a prostatic volume $\geq 60 \mathrm{~g}$, as measured by transrectal ultrasound (TRUS); IPSS $>8 ; Q \max \leq 15 \mathrm{ml} / \mathrm{s}$; and a voided urine volume $>150 \mathrm{ml}$.

The exclusion criteria were patients with significant co-morbidities and unfit for anesthesia; chronic renal impairment; urethral stricture; neurogenic bladder; chronic urinary retention; bladder pathology (e.g., bladder stone, bladder tumor); a history of urethral, bladder neck, or prostatic surgery; and prostate or bladder cancer.

Patients were randomly assigned using a computer software to group 1, included patients who underwent HoLEP enucleation of prostate and group 2, included patients who underwent bipolar resection of the prostate (Fig. 1).

Fifty-five patients were eligible for our selection criteria underwent HoLEP procedure and completed the study (group $1=55$ patients). Group 2 included 59 patients who were eligible for our selection criteria, underwent bipolar resection of the prostate and completed our follow-up protocol (group $2=59$ patients).

In both groups, the patients were evaluated before surgery by reviewing the results of a standard complete general and urological evaluation, along with their IPSS, urinary tract symptoms (e.g., voiding symptoms, storage symptoms, hematuria), and previous surgical and medical histories.

Patients also underwent general, abdominal, and local examinations of the external genitalia in addition to a focused neurological examination and a digital rectal examination (DRE). Furthermore, all patients had the following laboratory tests: complete urine analysis; urine culture and sensitivity, if needed; prostate-specific antigen (PSA); routine preoperative laboratory investigations (CBC, blood urea, serum creatinine, liver functions, and coagulation profile); and serum electrolytes (sodium, potassium). Imaging studies were also ordered, in the forms of abdomen and pelvic ultrasound, to assess PVR urine; TRUS to assess prostate size; and uroflowmetry to determine $Q$ max, pattern of voiding, voided volume, and flow time.

\section{Surgical procedures}

\subsection{HoLEP}

The HoLEP procedure was performed as described by Gilling et al. (2.0 J, $30 \mathrm{~Hz}, 80 \mathrm{~W}$, using a 550-um laser fibers [SphinX $100 \mathrm{~W}$ Holmium-YAG laser, LISA Laser Products, Katlenburg-Lindau, Germany]), $30^{\circ}$ lens system, and an Olympus camera head connected to a monitor. During the HoLEP, saline was used as an irrigation fluid. The prostatic lobes were dissected away from the prostatic capsule in exactly the same plane in which the surgeon's index finger moves during the performance of an open prostatectomy. Coagulation of the bleeding arteries was performed by defocusing the laser fiber. Morcellation was done after complete enucleation of the adenoma using a Piranha morcellator.

\subsection{Bipolar resection of the prostate}

The procedure was performed using a Karl Storz 26 Fr. Resectoscope with continuous wash, an adapted $30^{\circ}$ lens system, and an Olympus camera head connected to a monitor. Saline $\mathrm{NaCl} \%$ was used for irrigation with a bipolar generator (EMED spectrum, EU) set at $350 \mathrm{~W}$ (cutting mode) and $120 \mathrm{~W}$ (coagulation mode).

\section{Outcomes}

\subsection{Intraoperative and perioperative outcomes}

The data collected for measuring the intraoperative and perioperative outcomes included operating time, intraoperative hemoglobin level drop (preoperative hemoglobin 


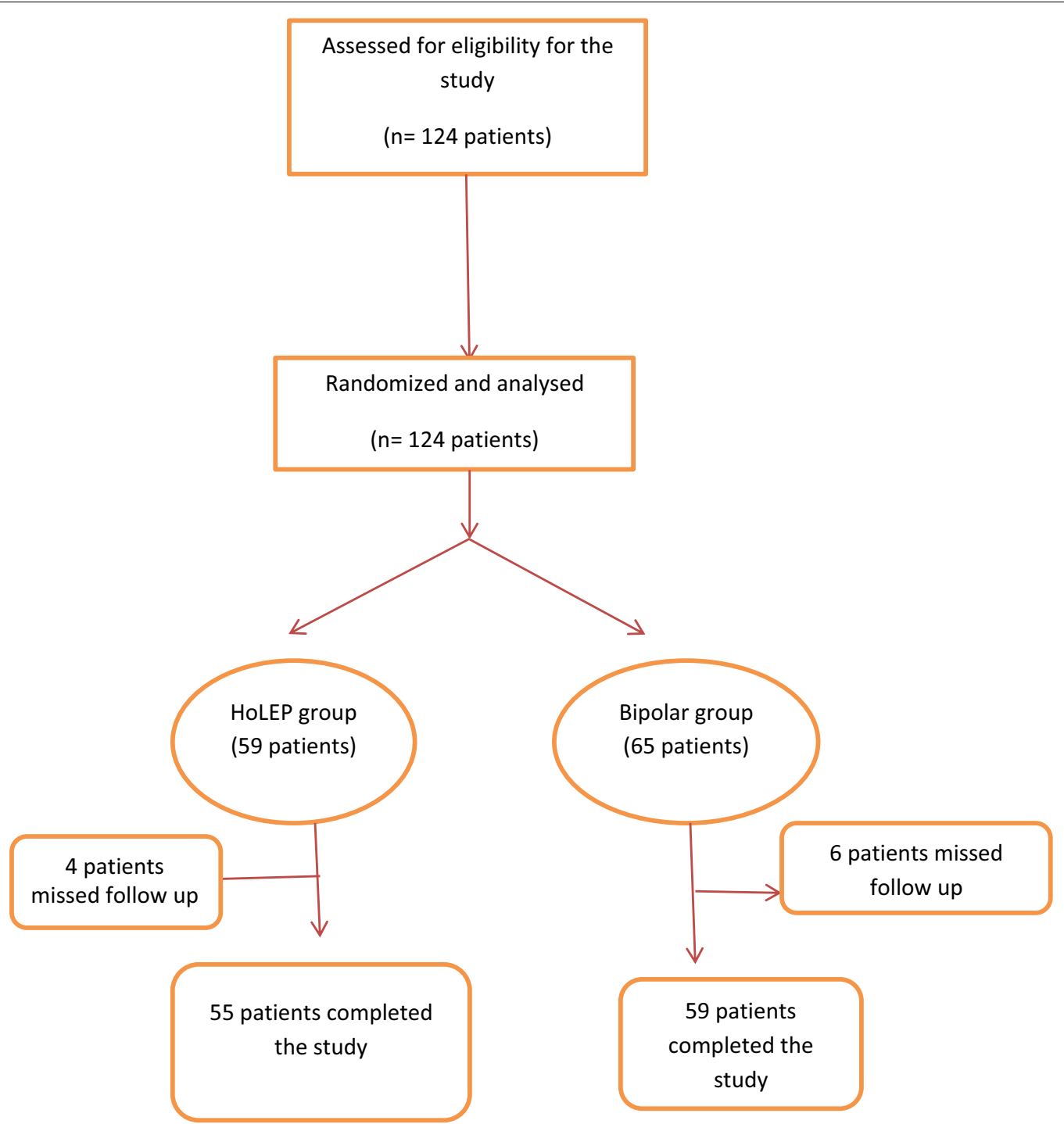

Fig. 1 Consort diagram

level-postoperative hemoglobin level), blood transfusion rate, resected tissue weight, length of hospital stay, duration of catheterization, and hemoglobin levels.

\subsection{Primary outcome}

To determine the primary outcome, the data collected included the recordings of IPSS and $Q$ max at $1,3,6,12$, and 24 months after surgery.

\subsection{Secondary outcomes}

For secondary outcomes, the data collected included recordings of PVR urine volume at 6 and 12 months after surgery.

\subsection{Follow-up}

The follow-up data collected included recording IPSS and $Q$ max scores at 1, 3, 6, 12, and 24 months and PVR urinary volume at six and 12 months. In addition, any adverse events, such as the development of urethral stricture, urinary incontinence, and necessity for reoperation, retrograde ejaculation, or urinary tract infection, were recorded.

\section{Statistical analyses}

The collected data, including history, physical examination, laboratory results, and outcome measures, were coded, entered, and analyzed using Microsoft Excel. The data were then imported into Statistical Package for the 
Social Sciences (SPSS) version 20.0 (IBM Corp. Armonk, NY, USA) for analysis.

The quantitative group results were represented as mean $\pm S D$, and the qualitative data were represented as numbers and percentages. A Chi-squared test (X2) was used to test the differences for significance, and an association of qualitative variable $t$ test was used to assess the differences between quantitative independent groups. The $P$ value was set at $<0.05$ for a significant result and at $<0.001$ for a highly significant result.

\section{Sample size calculation}

The sample size was calculated using G*Power version 3.1.9.4 and with a test family ( $\mathrm{t}$ tests), type of power analysis (a priori: compute required sample size-given $\alpha$, power, and effect size), input parameters, effect size $=0.82, \alpha$ error $=0.05$, power $(1-\beta)=0.8$, and assuming the allocation ratio $N_{1} / N_{2}=1$. The resulting output parameters indicated a sample size of 50 patients for each group. This yielded a total sample size of 100 patients.

\section{Results}

No statistically significant differences existed between the two groups regarding the preoperative parameters (Table 1).

Operative time was significantly shorter in the bipolar group, while the resected tissue weight was significantly higher in the HoLEP group.

The HoLEP group had significantly shorter postoperative catheter durations and hospital stays compared to the bipolar group.

Regarding postoperative loss in $\mathrm{HB}$ and Na levels, there were no statistically significant differences between the two groups (Table 2).

Preoperative IPSS scores showed statistically insignificant differences between both groups (Table 1).

As regard postoperative IPSS scores, there was no statistically significant difference between both groups at first month and 24 months postoperative.

Table 1 Preoperative data in both groups

\begin{tabular}{lccc}
\hline & $\begin{array}{c}\text { Bipolar-TURP } \\
\text { (mean } \pm \text { SD) }\end{array}$ & HoLEP (mean \pm SD) & $P$ \\
\hline Age (years) & $64.12 \pm 6.9$ & $63.79 \pm 5.6$ & 0.698 \\
IPSS & $25.05 \pm 4.10$ & $24.02 \pm 3.56$ & 0.114 \\
Prostate size by TRUS (g) & $75.02 \pm 9.23$ & $74.5 \pm 9.75$ & 0.698 \\
PVR (ml) & $157.75 \pm 32.4$ & $164.04 \pm 31.2$ & 0.392 \\
Q max (ml/s) & $6.58 \pm 2.1$ & $6.69 \pm 2.4$ & 0.623 \\
\hline
\end{tabular}

Table 2 Intra- and perioperative parameters

\begin{tabular}{lccc}
\hline & Bi-TURP & HoLEP & $P$ \\
\hline Resected tissue weight $(\mathrm{g})$ & $29.87 \pm 3.67$ & $39.66 \pm 4.24$ & 0.007 \\
Operation time $(\mathrm{min})$ & $51.39 \pm 7.89$ & $84.04 \pm 5.98$ & 0.005 \\
Postoperative HB $(\mathrm{g} / \mathrm{dl})$ & $11.19 \pm 1.02$ & $11.33 \pm 0.87$ & 0.12 \\
Loss of HB $(\mathrm{g})$ & $0.54 \pm 0.31$ & $0.49 \pm 0.12$ & 0.11 \\
Postoperative Na (mmol/L) & $139.55 \pm 1.02$ & $139.84 \pm 1.46$ & 0.12 \\
Loss in Na & $2.01 \pm 0.34$ & $2.03 \pm 0.05$ & 0.14 \\
Postoperative irrigation $(\mathrm{L})$ & $4.87 \pm 1.32$ & $4.42 \pm 1.13$ & 0.09 \\
Hospital stay (h) & $35.9 \pm 2.87$ & $33.55 \pm 1.9$ & 0.016 \\
Catheter duration (h) & $59.97 \pm 4.97$ & $24.55 \pm 1.33$ & 0.001 \\
\hline
\end{tabular}

$P$ value was set at $<0.05$ for a significant result are indicated in italic

However, IPSS scores at 3, 6 and 12 months postoperative were statistically significant different between both groups in favor of HoLEP group (Table 3).

Regarding preoperative and postoperative $Q \max$, there was a statistically significant difference between them in both groups. However, there were no statistically significant differences between both groups regarding $Q$ max at $1,3,6,12$, and 24 months. However, there were no statistically significant differences between both groups regarding postoperative PVR at 6 and 12 months (Table 3).

There were no statistically significant differences between the two groups regarding intra and postoperative complications. However, one patient in the bipolar group required a blood transfusion due to significant intraoperative bleeding. On the other side, three patients in the HoLEP group had bladder mucosal injuries during the morcellation, and prolonged catheterization was needed for two days.

Table 3 Follow-up data

\begin{tabular}{lccccc}
\hline & Bi-TURP & N & HoLEP & N & P \\
\hline IPSS at 1 month & $6.1 \pm 0.84$ & 55 & $5.9 \pm 0.89$ & 55 & 0.362 \\
IPSS at 3 months & $5.67 \pm 0.92$ & 55 & $5.12 \pm 0.5$ & 55 & 0.004 \\
IPSS at 6 months & $5.88 \pm 0.71$ & 55 & $5.32 \pm 0.49$ & 55 & 0.002 \\
IPSS at 12 months & $5.91 \pm 0.73$ & 55 & $5.28 \pm 0.41$ & 55 & 0.003 \\
IPSS at 24 months & $5.83 \pm 0.88$ & 48 & $5.87 \pm 0.97$ & 49 & 0.855 \\
Q max at 1 month & $24.44 \pm 2.75$ & 55 & $24.9 \pm 2.11$ & 55 & 0.351 \\
Q max at 3 months & $24.6 \pm 2.55$ & 55 & $25.05 \pm 2.22$ & 55 & 0.252 \\
Q max at 6 months & $24.22 \pm 2.9$ & 55 & $24.87 \pm 2.43$ & 55 & 0.154 \\
Q max at 12 months & $24.15 \pm 2.59$ & 55 & $24.95 \pm 1.93$ & 55 & 0.02 \\
Q max at 24 months & $24.0 \pm 2.33$ & 48 & $24.9 \pm 2.49$ & 49 & 0.091 \\
PVR at 6 months & $25.55 \pm 2.33$ & 55 & $24.45 \pm 4.55$ & 55 & 0.125 \\
PVR at 12 months & $29.43 \pm 1.95$ & 55 & $27.55 \pm 3.95$ & 55 & 0.088 \\
\hline
\end{tabular}

$P$ value was set at $<0.05$ for a significant result are indicated in italic 
Patients who suffered from urge incontinence in both groups where improved within two months using antimuscarinic drugs only. One patient in the HoLEP group developed postoperative total incontinence, mostly due to an injury of the external urinary sphincter, and was managed by using a penile clamp after refusing implantation of an artificial sphincter (Table 4).

\section{Discussion}

$\mathrm{BPH}$ is a common disease affecting aging males [9]. TURP had been considered the standard surgical procedure for the management of $\mathrm{BPH}$, as it has good surgical outcomes [6]. However, significant technical improvements during the past decade have reduced the intraoperative and postoperative adverse events associated with TURP, although there are still some concerns regarding complications such as bleeding, TUR syndrome, and urethral strictures [10].

HoLEP represents an attractive alternative to standard TURP because HoLEP has many advantages, including less blood loss, low postoperative irrigation, shorter catheterization times, and shorter hospital stays. HoLEP is safe to use in treating patients at risk of bleeding, such as those on anticoagulants or with coagulopathy [11]. The normal saline solution used for irrigation during the HoLEP procedure reduces the risk of TUR syndrome [12].

In a randomized study, Chen et al. compared HoLEP and bipolar TURP [13]. The study included 280 patients, and no significant differences were noted between both groups regarding IPSS and $Q$ max after 2 years; however, less bleeding, shorter catheterization times, and fewer hospital stays were reported in the bipolar group [13].

In the present study, there were no significant differences between both groups regarding preoperative data, including IPSS score, prostate size, and $Q$ max. The operative time was longer in the HoLEP group (including the morcellation time) than in the bipolar

Table 4 Intra- and postoperative complications

\begin{tabular}{llll}
\hline & Bi-TURP & HoLEP & $\boldsymbol{P}$ \\
\hline Intraoperative bleeding & $1(1.815 \%)$ & $0(0.0 \%)$ & 0.7 \\
Blood transfusion & $1(1.815 \%)$ & $0(0.0 \%)$ & 0.7 \\
Capsular injury & $7(12.72 \%)$ & $6(10.9 \%)$ & 0.78 \\
Bladder injury & $0(0.0 \%)$ & $3(5.45 \%)$ & 0.15 \\
UTI & $5(9.09 \%)$ & $4(7.27 \%)$ & 0.45 \\
Urge incontinence & $8(14.54 \%)$ & $10(18.18 \%)$ & 0.67 \\
Total incontinence & $0(0.0 \%)$ & $1(1.8 \%)$ & 0.55 \\
\hline
\end{tabular}

TURP group $(84.04 \pm 5.98 \mathrm{~min}$ vs. $51.39 \pm 7.89 \mathrm{~min}$, respectively; $P=0.005)$. However, Amr et al. reported longer operative times for a HoLEP group compared to a bipolar TURP group (96.1667 min vs. $81.2500 \mathrm{~min}$, respectively, $P=0.001$ ) [14].

In the present study, the longer operative time in the HoLEP group may be attributed to the morcellation time and the slow, careful enucleation of the prostatic apical tissue.

In the present study, more prostatic tissue was removed in the HoLEP group compared to the bipolar TURP group (39.66 \pm 4.24 g vs. $29.87 \pm 3.67$, respectively, $P=0.007)$. Amr et al. reported less prostatic tissue removed in their HoLEP than in their bipolar TURP group (55.9667 vs $65.6667 \mathrm{~g}$, respectively, $P=0.05$ ) [14].

No significant differences existed between both groups regarding intraoperative blood loss and blood transfusion rate. We also did not record any case of TUR syndrome in the bipolar group.

Only one case in the bipolar TURP group required a blood transfusion, and no cases in the HoLEP group required a blood transfusion. This may be explained by lasers causing deeper coagulative necrosis and subsequently less bleeding. The resection during the HoLEP procedure also occurs at the capsular level, thus the prostatic vessels are opened less frequently, unlike the bipolar resection that opens the vessels many times during the procedure [14].

At the 24-month follow-up examination, our study did not record any differences regarding IPSS and $Q$ max between the two groups. In their study, Fayad et al. reported that the 12-month postoperative IPSS scores were better in the HoLEP group compared to the bipolar group, and this difference was statistically significant [15].

Our study has a relatively small sample size, this may be explained by the long period of follow-up after surgery and the relatively poor compliance of many patients to complete the study. Further studies with longer follow-up and larger sample size are recommended to confirm our study results and conclusion.

\section{Conclusion}

HoLEP and bipolar resection of the prostate are effective and safe techniques used to treat patients with $\mathrm{BPH}$. Our study did not show significant differences between the two groups regarding $Q \max$ and IPSS scores at a 24-month of postoperative follow-up. Moreover, Bipolar resection of prostate is a cheaper technique with equivalent outcomes compared to HoLEP procedure. 


\section{Abbreviations}

$\mathrm{BPH}$ : Benign prostatic hyperplasia; HoLEP: Holmium laser enucleation of prostate; TURP: Transurethral resection of prostate; IPSS: International prostate symptom score; PVRU: Postvoid residual urine; HoLRP: Holmium laser resection prostate; TRUS: Transrectal ultrasound; HB: Hemoglobin.

\section{Acknowledgements}

Not applicable.

\section{Authors' contributions}

All authors have made a significant contribution to the findings and methods in the paper. AIA contributed to work idea, design of the work; MME contributed to the acquisition, analysis; AE performed interpretation of data; AAE drafted the work; EAS interpreted the data; AR helped with the acquisition and analysis; MSE drafted the work; MMA helped with the interpretation of data; LA contributed to the acquisition and analysis; MGH drafted the work; $\mathrm{AHI}$ contributed to data collection; EMI and MAH revised the manuscript. All authors have read and approved the final draft.

\section{Funding}

No funding was obtained for this study.

\section{Availability of data and material}

The datasets used and/or analyzed during the current study, available from the corresponding author on reasonable request.

\section{Ethics approval and consent to participate}

All procedures performed were in accordance with the ethical standards of the institutional and/or national research committee.

\section{Consent for publication}

Not applicable.

\section{Competing interests}

The authors declare that they have no competing interests.

\section{Ethical Approval}

Local institutional ethical approval was obtained (Zagazig University, faculty of medicine). Ethical Approval Number: ZU-IRB \#2529/26-1-2016.

\section{Informed Consent}

A written consent was taken from all participant after sufficient information about the study.

\footnotetext{
Author details

${ }^{1}$ Department of Urology, School of Medicine, Minia University, Minya 61111 , Egypt. ${ }^{2}$ Department of Urology, School of Medicine, Benha University, Benha University Hospital, Benha 61111, Egypt. ${ }^{3}$ Department of Urology, School of Medicine, Beni-Suef University, Beni-Suef University Hospital, Beni Suef 61111, Egypt. ${ }^{4}$ Department of Urology, School of Medicine, Zagazig University, Zagazig, Egypt. ${ }^{5}$ Glickman Urological and Kidney Institute, Cleveland Clinic, 9500 Euclid Ave, Q10, Cleveland, OH 44195, USA.
}

Received: 22 September 2020 Accepted: 19 January 2021

Published online: 01 February 2021

\section{References}

1. Gupta NP, Nayyar R (2013) Management of large prostatic adenoma: laser versus bipolar transurethral resection of the prostate. Indian J Urol 29(3):225-235

2. Wilson LC, Gilling PJ, Williams A et al (2006) A randomized trial comparing holmium laser enucleation versus transurethral resection of the prostate: results at 2 years. Eur Urol 60:569-573

3. Michalak J, Tzou D, Funk J (2015) HoLEP: the gold standard for the surgical management of BPH in the 21st century. Am J Clin Exp Urol 3:36-42

4. Starkman JS, Santucci RA (2005) Comparison of bipolar resection of the prostate with standard transurethral prostatectomy, shorter stay, earlier catheter removal and fewer complications. BJU Int 95:69-71

5. Tefekli A, Muslumanoglu AY, Baykal M et al (2005) A hybrid technique using bipolar energy in transurethral prostate surgery prospective, randomized comparison. J Urol 174:1339-1343

6. Maheshwair PN, Joshi N, Maheshwari RP (2013) Best laser for prostatectomy in the year 2013. Indian J Urol 29(3):236-243

7. Gilling PJ, Kennett K, Das AK et al (1998) Holmium laser enucleation of the prostate (HoLEP) combined with transurethral tissue morcellation: an update on the early clinical experience. J Endourol 12:457-459

8. Kelly DC, Das AK (2012) Holmium laser enucleation of the prostate technique for benign prostatic hyperplasia. Can J Urol 19(1):6131-6134

9. Djavan B, Nickel JC, de la Rosette J et al (2002) The urologist view of BPH progression: results of an international survey. Eur Urol 41:490

10. Rassweiler J, Teber D, Kuntz R et al (2006) Complications of transurethral resection of the prostate (TURP) —incidence, management, and prevention. Eur Urol 50:969

11. Kim M, Lee H, Oh S (2013) Technical aspects of holmium laser enucleation of the prostate for BPH. Korean J Urol 54:570-579

12. Abdel-Hakim AM, Habib El, El-Feel AS (2010) Holmium laser enucleation of the prostate: initial report of the first 230 Egyptian cases performed in a single center. Urology 76:448-452

13. Chen YB, Chen Q, Wang Z et al (2013) A prospective, randomized clinical trial comparing plasmakinetic resection of the prostate with holmium laser enucleation of the prostate based on a 2-year follow-up. J Urol 189:217-222

14. Amr SF, El Sheikh MG, Zakaria T, Elfottoh HA, Alsergany R (2011) Holmium laser enucleation versus bipolar resection of the prostate: a prospective randomized study. Which to choose? J Endourol 25:1347-1352. https:// doi.org/10.1089/end.2011.0059

15. Fayad AS, Elsheikh MG, Zakaria T, Elfottoh HA, Alsergany R, Elshenoufy A, Elghamarawy H (2015) Holmium laser enucleation of the prostate versus bipolar resection of the prostate: a prospective randomized study. "Pros and Cons". Urology. https://doi.org/10.1016/j.urology.2015.08.004

\section{Publisher's Note}

Springer Nature remains neutral with regard to jurisdictional claims in published maps and institutional affiliations.

\section{Submit your manuscript to a SpringerOpen ${ }^{\circ}$ journal and benefit from:}

- Convenient online submission

- Rigorous peer review

- Open access: articles freely available online

- High visibility within the field

- Retaining the copyright to your article

Submit your next manuscript at $\boldsymbol{\nabla}$ springeropen.com 